\title{
Issues for deep level models of bulk damage to silicon detectors
}

\author{
G. Hall
}

Blackett Laboratory, Imperial College

London SW7 2AZ, UK.

g.hall@ic.ac.uk

\section{Abstract}

A brief review of the current data is given and some of the major unresolved issues in the understanding of radiation damage to silicon detectors are highlighted, concentrating mainly on damage to the silicon bulk. The importance of experimental tests of the deep level models being developed is stressed as a key to further progress.

\section{Introduction}

In view of the operation of the CERN Large Hadron Collider to generate $14 \mathrm{TeV}$ centre of mass energy proton-proton collisions in the next decade and the well known problems of radiation damage to detectors and electronics, particularly in the vicinity of the interaction point, a large amount of experimental data has been gathered on the properties of silicon detectors after irradiation. Looking back a few years to the situation in October 1990, when a major preparatory meeting for the LHC was held [1], it is encouraging to observe the substantial progress which has been made. Machine operational scenarios, secondary particle fluences and radiation doses expected in the experiments are now quite well understood and particle distributions simulated in great detail [2,3]. Although many detector issues under discussion in 1990 have not been fully resolved, a significant quantity of new data has been accumulated [4] and the development of more realistic models of the microscopic changes occurring in detector material has taken place.

Although many changes take place during and after irradiation, for example at the surface of microstrip detectors, it seems that at least up to the particle fluences expected in inner tracking detectors at LHC, $\sim 10^{14} \mathrm{~cm}^{-2}$, most are not fundamental problems. The principal obstacle to long term operation of silicon detectors at LHC arises from damage to the bulk of the material which alters the effective doping of the material and thus operational voltages. In view of the large amount of data now available and models under discussion, it is interesting to ask if the phenomena are understood in a truly quantitative way. If so, one can also ask about the potential for production of radiation harder detectors, a topic which has in the past seemed more an ideal than a possible reality. 


\section{Experimental results}

In 1990, the dependence of damage on particle type was already quite well understood, for example calculations of Van Ginneken [5] used Non-Ionising Energy Loss (NIEL) as a means to infer the rate of damage by particles of different type and energy. More recently, concern about possible enhanced damage by low energy pions in inner tracking detectors led to new calculations of the effects of pions [6]. Resonance production in pion-nucleon scattering around $300 \mathrm{MeV} / \mathrm{c}$ could enhance displacement damage. Measurements are not fully conclusive but any increase in damage appears to be limited to not more than $20-30 \%$ compared to $1 \mathrm{MeV}$ neutrons.

Although caution must be used, for example in extrapolating from low energy beta electrons, or photons, to much higher energies, it seems that the NIEL hypothesis is in general accord with observation. Only $15-25 \mathrm{eV}$ of recoil energy is required to displace an atom from its lattice site, but this requires an electron with a kinetic energy of about $200 \mathrm{keV}$. Higher energy electrons create multiple displacements but only above several $\mathrm{MeV}$ do damage clusters occur, due to the removal of a silicon atom with enough kinetic energy to generate further displacements. Heavy particles generate clusters at lower kinetic energy and most irradiations have been carried out with hadrons and related to $1 \mathrm{MeV}$ neutrons for convenience of comparison. The principal observations are summarised below.

Diode leakage currents increase in linear proportion to particle fluence. After high energy hadron irradiation the volumetric current density $\left(\mathrm{JV}_{\mathrm{V}}\right)$ increases with fluence $(\Phi)$ as $\mathrm{J}_{\mathrm{V}}=\alpha \Phi$, with $\alpha \approx 5-10 \times 10^{-17} \mathrm{~A} \cdot \mathrm{cm}^{-1}$. The damage constant for photons is much smaller, $\alpha \approx 2 \times 10^{-23}$ A.cm-1. Although the linear dependence is apparently trivial, it is not easy to explain. It suggests a relationship between leakage current and the number of clusters since, in more detailed models of the defect evolution, most of the defect concentrations evolve in a non-linear fashion. It may seem self-evident that clusters contribute strongly to leakage currents, but this is a barrier to quantitative understanding since the Shockley-Read-Hall (SRH) theory of generationrecombination gives no means of calculation.

Annealing of bulk damage is important and must be taken into account since most irradiations are carried out at much higher fluxes than will be encountered in experiments. Leakage currents are observed to decrease by factors of 2-3. The current continues to be sensitive to temperature and can be reduced considerably by relatively modest cooling. The dependence is usually parameterised as $\mathrm{JV}^{\sim} \mathrm{T}^{2} \exp (-\mathrm{E} / \mathrm{kT})$. The $\mathrm{T}^{2}$ pre-factor does not have a big influence on fits to the data and simple SRH theory is usually taken to imply that $\mathrm{E}=\mathrm{E}_{\mathrm{g}} / 2$, with $\mathrm{E}_{\mathrm{g}}=1.1 \mathrm{eV}$, the silicon band gap. Experimental measurements consistently give $\mathrm{E}>0.55 \mathrm{eV}$ with $\mathrm{E} \approx 0.65 \mathrm{eV}$ more typical.

During irradiation there are changes in the effective doping concentration $\left(\mathrm{N}_{\mathrm{eff}}\right)$ of the bulk material. After sufficient fluence of heavy particles, the bulk silicon, which is normally n-type, is observed to invert and behave as p-type, under bias. A typical fluence of $1 \mathrm{MeV}$ neutrons to bring this about is $\Phi_{\text {inv }} \sim 2 \times 10^{13} \mathrm{~cm}^{-2}$; more detailed studies as a function of starting material resistivity [7] have parameterised the dependence on initial doping concentration $\left(\mathrm{N}_{0}\right)$ as $\Phi_{\text {inv }} \approx 19[\mathrm{~cm}] \times \mathrm{N}_{0}$.

Long term annealing is as important as short term effects. At ambient temperature there is an initial recovery of donors (or acceptor reduction) but later a slow increase of acceptor density is observed ("anti-annealing")[8]. The effect is especially marked after 
high fluence irradiations and leads to a need to operate the detectors at higher voltage to maintain full depletion and sufficiently rapid charge collection. Cooling to below about $5^{\circ} \mathrm{C}$ has been observed to control this effect but at the expense of the initial beneficial anneal, during which some of the damage is apparently repaired. The optimum temperature appears to be in the range $\sim 0^{\circ} \mathrm{C}$. This has been established by experiment but there is no theoretical explanation.

In addition to bulk damage, a large number of surface related effects have been observed, many of which have been studied in detail although dependence on the design and manufacturing process are inevitable so general conclusions become difficult to draw. A significant decrease in interstrip resistance on the junction side of microstrip detectors can become particularly important after type inversion if detectors are not significantly overdepleted. Increases in interstrip capacitance have been observed, although at the high frequencies where LHC electronics will operate the magnitude of the change can be limited with careful design. In double sided microstrip detectors it has been demonstrated that both field plate and p-stop isolation techniques work satisfactorily. Concerns about higher voltages have led to a preference for p-stop isolation since micro-discharges at field plates are observed to lead to premature breakdown. Integrated resistors and capacitors are quite feasible under LHC conditions, especially using polysilicon as the resistor material. The use of silicon nitride, in addition to oxide, as the capacitor dielectric appears to lead to greater reliability and fewer breakdown problems with no major drawbacks.

Much work is underway as part of the detector prototyping for ATLAS, CMS, HERA-B, etc to define the LHC microstrip detectors. Many of the problems would become significantly simpler if high voltage operation were not mandatory. Since the need for this originates in the doping changes, it is clear that better understanding of the origin of bulk damage is a potential key to improved detectors in many respects.

\section{Models of bulk damage in silicon}

Although an improved understanding of the origin of silicon bulk damage remains an intellectual challenge, one can question the need for persistence in developing a microscopic model, if only because investigations in this field have been underway for thirty years or more and the full solution to the problem may seem remote. Without a model we can already explain most of the dependence of damage on particle type and energy and are able to find pragmatic recipes for long term operation.

However, the uncertainty in future extrapolations is of great concern, especially since some detectors, such as pixels close to the interaction point, will certainly not survive indefinite operation at LHC, even with cooling. Even an imperfect microscopic model would lead to a considerable gain in confidence for long term LHC operation if it clarified, for example, the value of $\mathrm{N}_{\text {eff }}$ and evolution with fluence. In addition there is now much data which a model should explain, such as resistivity in thermal equilibrium conditions, the absolute value of leakage current and its evolution with fluence, temperature dependence of current, I-V characteristics, anti-annealing. A successful model would also define the dependence of damage on environment and, most important, the possibilities for detector hardening. 
The fundamental origin of bulk damage can certainly be traced to energy levels within the silicon band gap, introduced as a result of damage to the crystalline symmetry of the lattice. Some of the most prominent defect complexes have been identified in many studies, but there are a large number of less well understood defects and some of these may be implicated in the observations. In addition, the importance of damage clusters, whose internal structure is not clear, could limit fundamental understanding.

The primary defects generated by displacements are lattice vacancies (V) and silicon interstitial atoms (I). Both of them are mobile at room temperature, with different speeds. In electron and photon irradiations, vacancies and interstitials migrate through the crystal, eventually becoming trapped at capture sites. In contrast, high energy heavy particles frequently displace an atom from its lattice site with a high recoil energy. The recoil atom produces a cascade with many interactions, some of which also produce energetic secondary recoils. Each of these terminates in a damage cluster which contains a high density of interstitial and vacancy pairs as well as significant disorder. In the interior of the cluster, where initial concentrations of vacancies and interstitials are high, direct recombination occurs and most I-V pairs annihilate on a very short timescale.

Interstitial atoms diffuse out from clusters more rapidly than vacancies and defect interactions take place in the cluster volume. During this period divacancy $\left(\mathrm{V}_{2}\right)$ production occurs; obviously electrons and photons produce fewer "primary" divacancies than heavy particles. Divacancies are not mobile and thus remain trapped in the cluster region. Interactions of defects with impurities are initially rare because of the size of the cluster (linear dimensions $\sim 100 \AA$ ). Later, trapping by impurities, such as oxygen and carbon, leads to formation of stable defect complexes.

Recently, from experimental measurements using DLTS, primary introduction rates (I, $\mathrm{V}$ and $\mathrm{V}_{2}$ ) have been inferred. Using these as input a numerical model of the evolution of complex defects has been constructed. Many complexes have been well characterised in experimental studies by materials scientists and parameters such as energy levels and charge states are known. The model offers a foundation stone for understanding the data.

\section{Deep acceptor model}

A numerical estimate of defect concentrations is one essential piece of information to construct a model. A second is a reasonably rigorous application of semiconductor physics to predict the consequences of traps. Both "shallow" and "deep" levels occur; shallow levels are found closer to the band edges. In silicon shallow acceptors and donors, such as boron and phosphorus, are used to dope the material since they generate states which are a few meV from either the conduction band (phosphorus) or valence band (boron) and are ionised at room temperature, providing a free charge carrier. Deep levels can exist in different charge states depending on conditions in the material and the behaviour of the trap as a donor or acceptor.

Most deep levels have two charge states, so an acceptor is negatively charged when occupied by an electron and neutral when empty, while a donor is positively charged when unoccupied but neutral otherwise. In unbiased material, where thermal 
equilibrium holds, the occupation of a state depends on Fermi-Dirac statistics and is calculable if the concentration of states and their energy levels are known. Thus the resistivity of irradiated silicon wafers, which can be directly measured by probing, can be estimated from the model. In biased material the situation is more complex because the charge state depends on the trap energy level but also on the density of free carriers. It is necessary to solve Poisson's equation for the electric field, satisfy current continuity conditions and include correct occupancy statistics, which usually makes numerical solution essential.

Such calculations were first carried out by Watts and collaborators [9]. They hypothesised, to simplify, that after high particle fluences bulk silicon was intrinsic and assumed a single acceptor level at the middle of the energy gap. Both assumptions are justified by experimental data, since highly irradiated silicon does seem to become almost intrinsic in thermal equilibrium, while under bias it behaves as strongly p-type. Using the measured value of current density with fluence and occupancy from Shockley-Read-Hall statistics, they calculated the depletion voltage, and thus effective doping concentration, as a function of fluence.

There is only one free parameter, the introduction rate of the acceptor state; which was adjusted to match the data. It is possible to explain the observed behaviour of silicon diodes constructed on p-type and n-type substrates after neutron irradiation surprisingly well (fig. 1). If the assumption that the acceptor is not at mid-gap is dropped, the required introduction rate can be plotted as a function of the trap energy level [10].

Of course the model does not require that only a single deep level be present, nor that only acceptors are created but it should somehow eventually lead to a natural explanation of why acceptors dominate. However it already suggests that an explanation based on deep levels generated during irradiation is likely. The essence of the hypothesis was verified by observing the depletion behaviour of diodes under illumination; it is clearly observed that irradiated detectors behave differently to nonirradiated ones in a manner consistent with deep traps being filled by photo-generated carriers [11].

\section{Alternatives to the deep acceptor model.}

Prior to the deep acceptor hypothesis, the most plausible explanation of doping changes under irradiation was that the phosphorus dopant was deactivated electrically by capturing a lattice vacancy. Introduction of a shallow acceptor was also assumed, to cause type inversion once the phosphorus was exhausted. The combination of donor removal and acceptor creation provides a satisfactory parameterisation of much of the data but does not explain anti-annealing effects without a better understanding of the nature of the defects present in the silicon.

Despite being initially attractive, several problems are posed by this picture. There is no direct evidence for sufficient phosphorus removal to explain inversion and recent DLTS studies show a relatively low removal rate; other measurements can easily be explained by the deep acceptor hypothesis. There is no clear candidate for the shallow acceptor state and it is not obvious why apparently few donors are introduced. Another strong argument against this idea is that it oversimplifies some important physics, 
since the calculation described above demonstrates that deep levels, which are known to be present in the material, must be accounted for in the theory.

\section{Microscopic explanation of bulk damage}

The numerical model of complex defect evolution was originally developed to explain optical absorption data at infra-red wavelengths after irradiation by $2 \mathrm{MeV}$ electrons [12]. Characteristic absorption lines correspond to the presence of known defect centres and the concentrations of several species were determined as a function of electron fluence using samples of Czochralski and float-zone material, where initial impurity concentrations had been accurately determined. The model required some modifications to be applicable to high resistivity detector material, in particular to include the presence of phosphorus.

Reaction rates are controlled by the concentration of impurities and defects and their capture radii. Vacancies are principally captured by oxygen and so much is present $\left(\sim 10^{16} \mathrm{~cm}^{-3}\right)$ compared to phosphorus $\left(\sim 10^{12} \mathrm{~cm}^{-3}\right)$, that phosphorus removal is limited. Similarly, high carbon concentrations (also $\sim 10^{16} \mathrm{~cm}^{-3}$ ) provide the major sink for interstitials. Measured introduction rates of interstitials, vacancies and primary divacancies from electron and neutron irradiations were used to predict defect concentrations in high resistivity detector material during neutron and gamma irradiation. The main uncertainties arise from errors on primary introduction rates, of which there are few measurements, and the concentrations of oxygen and carbon, which have been independently checked using several techniques.

Two multi-vacancy oxygen complexes, $\mathrm{V}_{2} \mathrm{O}$ and $\mathrm{V}_{3} \mathrm{O}$, look interesting candidates for a deep-level acceptor, of which only $\mathrm{V}_{2} \mathrm{O}$ could cause type inversion since at fluences $\sim 2 \times 10^{13}$ n.cm ${ }^{-2}$ there are too few $\mathrm{V}_{3} \mathrm{O}$ defects to compensate the phosphorus. $\mathrm{V}_{3} \mathrm{O}$ might contribute to subsequent annealing.

\section{Detector leakage currents}

A correlation between leakage current and doping concentration is expected as acceptor occupancy, and consequently $\mathrm{N}_{\mathrm{eff}}$, is limited by the free electron density in the material, which is related to the leakage current. If the deep level model is complete and correct, it should be possible to explain the evolution of doping concentration and leakage current in a self consistent way. Shockley and Read [13] first calculated how traps in the band gap contribute to generation-recombination currents, which dominate in irradiated diodes. The situation is illustrated for the case of a two level acceptor or donor state in fig. 2. Each state communicates only with the conduction band and valence band.

In thermal equilibrium, emission and capture rates of electrons (or holes) are identical. This detailed balance allows the calculation of emission rates from a trap by the use of Fermi-Dirac statistics for occupancy. Under reverse bias, when the system is not in thermal equilibrium, it is assumed that emission rates are unchanged. Capture reactions are suppressed by the reduction of mobile carrier densities so leakage currents arise from emission processes. The occupancy of the trap is derived from the steady state condition of equal hole and electron generation rates. This leads to: 


$$
G=\frac{N_{t} \sigma_{n} \sigma_{p} v\left[n_{i}^{2}-n p\right]}{\sigma_{n}\left(n_{i} e^{\beta}+n\right)+\sigma_{p}\left(n_{i} e^{-\beta}+p\right)} \text { with } \beta=\frac{E_{t}-E_{i}}{k T}
$$

for the carrier generation rate. $N_{t}$ is the trap concentration, $n_{i}$ is the intrinsic carrier concentration, $\mathrm{n}$ and $\mathrm{p}$ are the electron and hole concentrations $\left(<<\mathrm{n}_{\mathrm{i}}\right.$ under reverse bias). The trap energy level is $E_{t}$ and the intrinsic level is $E_{i} ; k T$ is the Boltzmann factor and $\mathrm{v}$ is the thermal carrier velocity. A further simplification is achieved by assuming equality of $\sigma_{\mathrm{n}}$ and $\sigma_{\mathrm{p}}$, the electron and hole cross-sections:

$$
G \approx \frac{N_{t} \sigma u n_{i}}{\left(e^{\beta}+e^{-\beta}\right)}
$$

Usually it is claimed that traps close to the centre of the band gap dominate and thus the temperature dependence of $\mathrm{G}$ follows $\mathrm{n}_{\mathrm{i}}$. However, it should be noted that a state only a small distance from the mid-gap in sufficient concentration may lead to a different temperature dependence due to the properties of the exponentials, thus

$$
G \approx N_{t} \sigma v n_{i} e^{-\beta}
$$

It is interesting to ask if this provides the explanation for the observed temperature dependence and if it can be used to identify better states contributing to the doping changes.

Calculations of the leakage current and doping changes have been carried out [14]. and compared with measurements after gamma and neutron irradiation. It was found that, while gamma results can be predicted rather well, there is a large discrepancy in the leakage current of neutron irradiated diodes compared to the model, by approximately a factor 100, and that details of doping changes are rather poorly described. Since leakage current and effective doping are correlated, this leads one to question if a single mechanism is responsible.

\section{Communication between traps}

Since the deep level idea appears to be fruitful, it is tempting not to abandon it too quickly. Although clusters are not well understood, it may be a mistake to invoke their assumed internal complexity, as a "catch-all" explanation of anomalous results. Similarly, although unidentified traps could be responsible for the extra leakage current, the success of the model for gamma irradiated material, where point-like damage should be easiest to calculate, suggests that the basic ideas are sound. In addition, the magnitude of the discrepancy implies that any unidentified trap must be present in large concentration and it is surprising that no obvious candidate is apparent.

The discrepancy is probably linked to the clusters in neutron irradiated material. The disordered regions must retain significant order because divacancies are readily identifiable in high concentration in DLTS studies. If the material were locally amorphous, one might expect that the normal band structure would not be preserved and that defects inside clusters would not appear so clearly. It was pointed out that the high $V_{2}$ density in clusters could modify the normal SRH picture of generationrecombination [15]. The cluster introduction rate was estimated and compared with the $\mathrm{V}_{2}$ introduction rate. It suggests that 10-20 divacancies are present in each cluster, 
which is a region of volume $(\sim 100 \AA)^{3}$, and there is a possibility that some of them will be sufficiently close to exchange charges.

Recently, charge exchange reactions have been observed and enhanced diode currents attributed to this mechanism [16]. However, the communication was between two different defect types where reactions of the type $X^{0} Y^{-}<=>X^{-} Y^{0}$ can enhance the occupation of a state with a higher emission rate. Reactions of the type $X^{0} X^{-}<=>X^{-} X^{0}$ cannot have the same consequence. However, the divacancy is unusual as it is known to exist in four possible charge configurations: $\mathrm{V}_{2}{ }^{+}, \mathrm{V}_{2}{ }^{0}, \mathrm{~V}_{2}{ }^{-}, \mathrm{V}_{2}{ }^{+}$. The positive and double negative states have very high emission rates. It is therefore possible that the reaction $\mathrm{V}_{2}{ }^{0} \mathrm{~V}_{2}{ }^{0}=>\mathrm{V}_{2}-\mathrm{V}_{2}{ }^{+}$occurs, since the population of $\mathrm{V}_{2}{ }^{0}$ predominates by a large factor over other charge states, which will lead to an enhanced occupation of $\mathrm{V}_{2}{ }^{-}$and $\mathrm{V}_{2}{ }^{+}$and a higher carrier generation rate. Although other reactions are possible, they should be minor contributors to this effect.

The "effective" local density of $\mathrm{V}_{2}$ in the clusters, i.e. the fraction of them which do exchange charge is unknown. Leaving this as a free parameter, numerical calculation [15] suggests strongly that an enhancement of the leakage current by the observed factor is indeed possible. A second important consequence is that the population of negatively charged divacancies is enhanced by a similar factor. These acceptor states may then dominate over the other defect complexes and must be taken into account in the modelling of $\mathrm{N}_{\text {eff }}$ which could explain the discrepancy there also.

\section{Detector hardening}

If the deep level model is generally correct, key ingredients in possible radiation harder detectors may be oxygen and carbon since these are the dominant capture sites for vacancies and interstitials. High oxygen concentration suppresses phosphorus removal and encourages A-centre (VO) formation at the expense of $\mathrm{V}_{2} \mathrm{O}$ and $\mathrm{V}_{3} \mathrm{O}$. The A-centre is relatively harmless because of its distance in energy from mid-gap, so an increase in the initial oxygen concentration should be beneficial. In the case of carbon, it appears that either a significant increase or decrease in concentration may be beneficial. If carbon concentration is reduced, the accumulation of multi-vacancy complexes is reduced by the availability of more interstitials for recombination. Conversely, modelling suggests that higher carbon concentration can reduce the free vacancy population because of the importance of the reaction $\mathrm{I}+\mathrm{V}_{2}-\mathrm{V}$. To understand the relative importance of carbon, numerical simulations and accurate impurity concentration measurements are required.

However, if divacancy charge exchange is responsible for the high leakage current in neutron irradiated material there seems much less hope to harden the raw detector material. The temperature dependence of the leakage current in neutron irradiated material does seem suggestive of an association with the divacancy but further experimental tests of this idea are essential. .

To be complete a model of bulk damage ought also to provide an explanation of the phenomenon of anti-annealing, which remains less satisfactory. The most obvious mechanism is by release of vacancies during annealing of clusters, possibly to amalgamate with $\mathrm{V}_{2} 0$ to give $\mathrm{V}_{3} 0$. This immediately suggests that anti-annealing 
should not be significantly present after photon irradiation, which is supported by experiment [14]. However the exact origin of free vacancies released during annealing remains obscure and without this knowledge quantitative annealing behaviour is difficult to predict .

\section{Conclusions}

It is encouraging to note that the scientific method is still effective in the mature field of silicon radiation damage. The gathering of new data has allowed the elimination of hypotheses and motivated the construction of new models through which further considerable progress has been made. The deep level model appears to provide a key to understanding more deeply the origin of radiation damage in silicon but it must continue to be critically scrutinised and subjected to detailed experimental tests.

\section{Acknowledgements}

This article is not intended to be a full review of an area to which there have been many important contributions over many years. I apologise to those whose work has been inadequately represented here. I warmly thank Barry MacEvoy, Karl Gill and Steve Watts for valuable discussions.

\section{References}

[1] G. Jarlskog, D. Rein (eds), ECFA Large Hadron Collider Workshop proceedings, CERN 90-10 (1990)

[2] CMS Technical Proposal CERN/LHCC 94-38 (1994)

[3] ATLAS Technical Proposal CERN/LHCC 94-43 (1994)

[4] More complete references can be found in G. Hall. Nucl. Instr. \& Meths A368 (1995) 199-204.

[5] A. Van Ginneken. Fermilab Report FN-522 (1989)

[6] M Huhtinen \& P. Aarnio. Nucl. Instr. and Meths. A335 (1993) 508

[7] S. Bates. Talk given at 2nd International Conference on Large Scale Applications and Radiation Hardness of Semiconductors, Florence, May 1995.

[8] S. Sotthibandhu. Ph.D thesis, Imperial College, RALT-025 (1994).

[9] J. Matheson, M. Robbins, S. J. Watts. The effect of radiation induced defects on the performance of high resistivity silicon diodes. RD20/TN/36 (1994). To be published.

[10] J. Matheson, M. Robbins, S. Watts, G. Hall, B. MacEvoy. Nucl. Instr. and Meths. in print.

[11] B. MacEvoy, G. Hall and K. Gill. Defect evolution in irradiated silicon detector material. IC/HEP/95-8. Nucl. Instr. and Meths in print.

[12] G. Davies et al. Semicond. Sci. Technol. 2 (1987) 524-532

[13] W. Shockley and W. Read. Phys. Rev. 87 (1952) 835.

[14] B. MacEvoy. These proceedings.

[15] K. Gill et al. Divacancy clustering and the origin of leakage current in bulk damaged silicon detectors CMS/TN/96-17 (1996)

[16] A. Schenk andU. Krumbein. J. Appl. Phys 78 (1995) 3185. 


\section{Figure captions}

Fig. 1. Neff vs $1 \mathrm{MeV}$ neutron flux for $\mathrm{n}$ - and p-type silicon. Lines represent fits to data from the RD2 collaboration and symbols are from model calculations.

Fig. 2 Electron and hole emission and capture processes from a single isolated level.

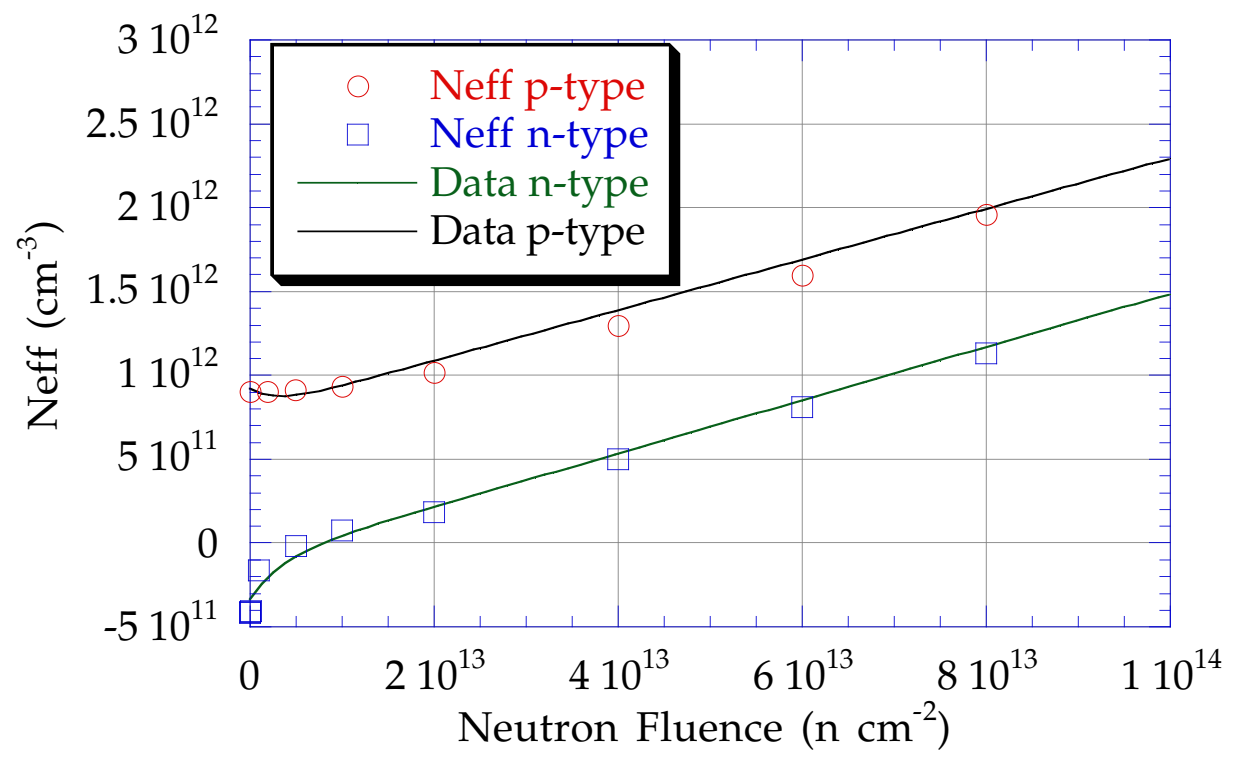

Fig. 1.

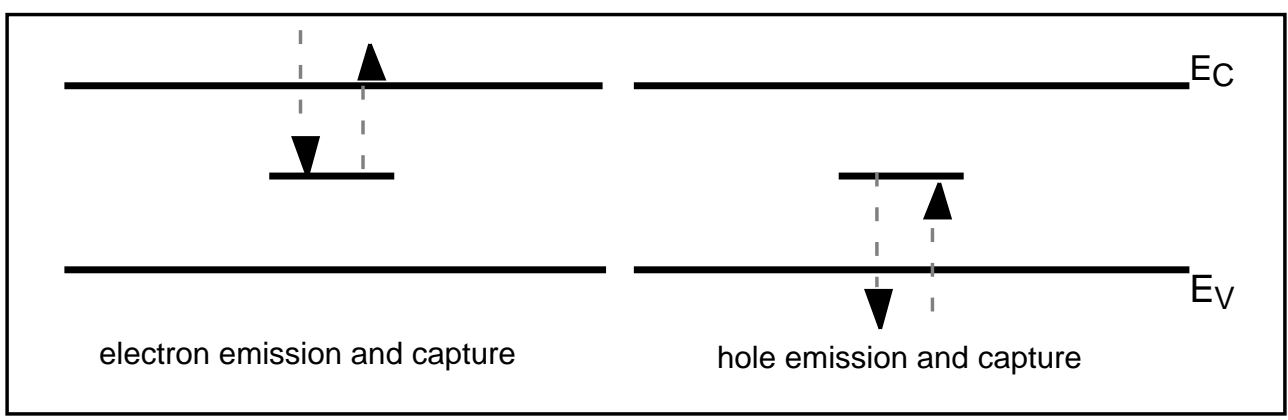

Fig. 2 